\title{
Do Somatic Mitochondrial DNA Mutations Contribute to Parkinson's Disease?
}

\author{
Joanne Clark, Ying Dai, and David K. Simon \\ Department of Neurology, Beth Israel Deaconess Medical Center, 330 Brookline Avenue, E/CLS-628, Boston, MA 02215, USA \\ Correspondence should be addressed to David K. Simon, dsimon1@bidmc.harvard.edu
}

Received 23 November 2010; Accepted 1 March 2011

Academic Editor: R. H. Swerdlow

Copyright () 2011 Joanne Clark et al. This is an open access article distributed under the Creative Commons Attribution License, which permits unrestricted use, distribution, and reproduction in any medium, provided the original work is properly cited.

A great deal of evidence supports a role for mitochondrial dysfunction in the pathogenesis of Parkinson's disease (PD), although the origin of the mitochondrial dysfunction in PD remains unclear. Expression of mitochondrial DNA (mtDNA) from PD patients in "cybrid" cell lines recapitulates the mitochondrial defect, implicating a role for mtDNA mutations, but the specific mutations responsible for the mitochondrial dysfunction in PD have been difficult to identify. Somatic mtDNA point mutations and deletions accumulate with age and reach high levels in substantia nigra (SN) neurons. Mutations in mitochondrial DNA polymerase $\gamma$ (POLG) that lead to the accumulation of mtDNA mutations are associated with a premature aging phenotype in "mutator" mice, although overt parkinsonism has not been reported in these mice, and with parkinsonism in humans. Together these data support, but do not yet prove, the hypothesis that the accumulation of somatic mtDNA mutations in SN neurons contribute to the pathogenesis of PD.

\section{Evidence for Mitochondrial Dysfunction in PD}

A large body of evidence suggests that mitochondrial dysfunction is a common pathological mechanism of neurodegenerative diseases including PD. There is a significant decrease in complex I activity of the mitochondrial electron transport chain and reduced immunohistochemical staining for complex I subunits in the substantia nigra (SN) from PD patients [1-4]. A recent meta-analysis of genome-wide expression data from SN neurons of patients with symptomatic and subclinical PD identified defects in mitochondrial electron transport, glucose utilization and sensing [5]. One key set of genes found in this study to be underexpressed in the brains of PD patients is under the control of the transcriptional coactivator peroxisome proliferator-activated receptor gamma coactivator 1-alpha (PGC-1 $\alpha$ ), a master regulator of mitochondrial biogenesis. Moreover, functional imaging like PET and functional magnetic resonance imaging (fMRI) show glucose metabolic defect in the frontal cortex in PD patients $[6,7]$. Together, these and other data provide clear evidence for a defect in mitochondrial metabolism in PD.

\section{Mitochondrial Dysfunction: Chicken or Egg?}

Although evidence for defective mitochondrial energy metabolism in PD is strong, such a defect theoretically could be a consequence or marker of neurodegeneration rather than a contributor to the pathogenesis of $\mathrm{PD}$. The most compelling evidence supporting a pathogenic role for mitochondrial dysfunction in PD comes from accidental human exposures to 1-methyl-4-phenyl-1, 2, 3, 6tetrahydrodropyridine (MPTP), which causes parkinsonism in human by inhibition of mitochondrial complex-I of the electron transport chain [8, 9]. MPTP also inhibits alpha-ketoglutarate dehydrogenase; therefore there was some debate as to whether or not the mechanism behind MPTP toxicity involved complex I inhibition [10]. This question has since been addressed by the subsequent discovery that chronic systemic administration of rotenone, a highly specific complex-I inhibitor, causes highly selective and progressive nigrostriatal dopaminergic degeneration and a parkinsonian phenotype in rats, further implicating mitochondrial complex I dysfunction in PD pathogenesis [11$13]$. 


\section{Origin of the Mitochondrial Complex-I Defect in PD}

The evidence cited above implicating a pathogenic role for mitochondrial complex I dysfunction in PD has led to great interest in understanding the origin of the complex I defect. Cytoplasmic hybrid (cybrid) cell lines created by transferring cytoplasmic contents including mitochondria from platelets or enucleated donor cells into cells experimentally depleted of endogenous mtDNA can be used to determine if a mitochondrial defect results from mtDNA mutations versus other causes (e.g., mitochondrial toxins or nuclear DNA mutations) [14]. Cybrids expressing mtDNA from idiopathic PD patients show reduced complex-I activity and increased susceptibility to $\mathrm{MPP}+$, suggesting that mtDNA encoded defects in PD $[15,16]$. Because cybrid cell lines share the same nuclear DNA background and differ only in the source of their mtDNA, these data implicate stable change in mtDNA (e.g., mutations) as a cause of the complex I defect in PD. In contrast, cybrid cell lines expressing mtDNA from subjects in the "Contursi" kindred who develop PD due to an autosomal dominant mutation in the $\alpha$-synuclein gene do not manifest complex I deficiency [17]. This suggests that a nuclear genetic mutation can cause PD in the absence of mtDNA mutations, and that mtDNA mutations are more likely to contribute to idiopathic PD than to less common forms of PD associated with known nuclear genetic mutations.

\section{Mitochondrial DNA and Classification of Mutations}

These data from cybrids implicating mtDNA mutations in the complex I defect in PD therefore led to the search for inherited mtDNA mutations in PD patients. There are three main classes of mtDNA mutations: ancient maternally inherited polymorphisms that have given rise to various mitochondrial haplogroups, evolutionarily recent maternally inherited potentially pathogenic mutations, and somatic mtDNA mutations that accumulate during the life of the organism [18]. A number of studies have linked certain mitochondrial haplogroups with increased or decreased risk of PD in various populations of differing ethnicity [1927]. Our laboratory found the G11778A mtDNA point mutation in a subunit of mitochondrial complex I in a family with maternally inherited parkinsonism and multisystem degeneration including nigral cell loss, demonstrating an association between an inherited mtDNA mutation and parkinsonism [28]. However, attempts to identify clearly pathogenic inherited mtDNA mutations in PD patients by direct sequencing of mtDNA have revealed that most $\mathrm{PD}$ patients lack such mutations [29-31]. The common 10398G variant of the NADH dehydrogenase 3 (ND3) gene (a subunit of mitochondrial complex I), present in up to $40 \%$ of Caucasians, has been reported to be present in a lower percentage of Caucasian PD patients compared to matched controls, suggesting a protective role in PD [32]. However, although a similar association was identified in another study [33], others have not replicated this result [27, 29].
Thus, although mtDNA point mutations have been found in rare families exhibiting parkinsonism [28, 34-39], clearly pathogenic mutations have not been identified in the vast majority of PD patients. Although a role of common mtDNA variants and rare pathogenic point mutations remains a possibility for some $\mathrm{PD}$ patients, these data led to the hypothesis that somatic mtDNA mutations, which may not be detectible by standard sequencing methods, might contribute to the complex I defect in PD.

\section{A Role for Somatic mtDNA Mutations}

The term "somatic mutations" (also known as acquired mutations) refers to DNA mutations that were not present in the germ cells that gave rise to the organism, but occurred later in development of the organism. Somatic mutations in mtDNA may take the form of single point mutations or small or large mtDNA deletions. mtDNA point mutations and mtDNA deletions can arise through separate mechanisms, although both types of mutation are now thought to be more commonly due to endogenous processes as opposed to exposure to exogenous agents $[40,41]$. Somatic mtDNA point mutations may arise in three ways: firstly, basesubstitution mutations caused by mitochondrial polymerase $\gamma$ infidelity $[42,43]$, and mitochondrial polymerase $\gamma$ replicating across damaged bases [40] may arise. Secondly, the proximity of mtDNA to ROS generated from the electron transport chain (ETC), as well as the lack of protective histones [44], may lead to oxidative damage to mtDNA, which can be highly mutagenic $[45,46]$. Indeed, levels of oxidative damage to mtDNA in the brain are much higher than levels of damage to nuclear DNA, particularly in elderly subjects [47]. It is possible that this is especially pronounced in the SN where dopamine metabolism, high levels of iron, and low levels of glutathione (an important antioxidant), may create an environment especially high in oxidative stress [48, 49]. Finally, although mitochondria lack nucleotide excision repair mechanisms, the mitochondrial genome is protected by base excision repair mechanisms [50]. However, a decline in the base excision repair mechanism has been observed with age [51].

A number of hypotheses have been put forward for how mtDNA deletions may occur: initially it was thought that strand slippage during replication of mtDNA molecules $[52,53]$ gave rise to deletions in mtDNA, but more recently a theory that mtDNA deletions are created during the repair of mtDNA double strand breaks has been proposed [41]. Conclusive experimental evidence for either theory is currently insufficient.

\section{Clonal Expansion of mtDNA Somatic Mutations}

The term heteroplasmy refers to the instance where mtDNA mutations affect only a proportion of the mitochondrial genome within a single cell, tissue, or organism. In contrast, the term homoplasmy is used when an mtDNA mutation affects all copies of the mitochondrial genome within 
a defined unit. Heteroplasmic somatic mutations may become functionally significant for two reasons: firstly, mtDNA mutations may undergo clonal expansion within individual cells. Clonal expansion of mtDNA mutations has been observed for both mtDNA deletions [54-56] and mtDNA point mutations [57]; however, the mechanisms behind clonal expansion are still under investigation. The high mtDNA content per cell and dynamism of the mitochondrial genome may be a contributing factor: each mitochondrion may contain several mitochondrial genomes, and each cell contains multiple mitochondria (the number of which varies according to the energy requirements of the cell), resulting in an estimated $10^{3}$ to $10^{4}$ copies of the mitochondrial genome per cell [58]. In addition, mitochondria are dynamic organelles and frequently undergo fusion and fission as well as frequent rounds of mtDNA replication $[59,60]$. This raises the possibility of clonal expansion of a particular mtDNA mutation through random genetic drift, which has been demonstrated experimentally after induction of "relaxed" mtDNA replication [61], which refers to replication of mitochondria in nondividing cells. In addition, theories of directed segregation have been proposed. Directed segregation has been demonstrated to select against mtDNA mutations [62] and seemingly paradoxically, to also select for mutant mtDNA via selection for advantageous replicative mechanisms [63]. A definitive answer to the mechanisms behind clonal expansion is not yet available. It is possible that there will be more than one mechanism and that this may vary depending on the mitotic or postmitotic nature of the cells [64].

\section{Aggregate Burden of Somatic Mutations and Failure of Functional Complementation}

Once a certain "phenotypic threshold" of clonal expansion has been reached (around 50\%-95\% of mtDNA copies per cell depending on the mutation, cell type, and other factors; [65-69] a deleterious mutation may lead to impaired mitochondrial function [70, 71] as beyond that level, functional mitochondrial complementation may no longer provide restoration of functional ETC complexes [72]).

To elaborate further, the term "functional mitochondrial complementation" refers to the exchange of genetic information between mitochondria to alleviate a respiratory deficit caused by the mutation of an ETC gene in one of the mitochondria [73]. mtDNA is packaged in genetically autonomous mtDNA-protein complexes called nucleoids [74-76], these are mobile elements that are located throughout the mitochondrial genome [77] and include the transcription factor TFAM, mitochondrial polymerase $\gamma$, and the mitochondrial helicase Twinkle [78]. Nucleoids work to maintain heterologous mtDNA as different populations, preventing intermixing between mtDNA populations [79]. However, complementation can occur in stably separate nucleoid populations through transcomplementation by transcripts and polypeptides from nonmutant mitochondria freely diffusing through the mitochondrial matrix and becoming translated or assembled into ETC complexes, leading to a restoration of mitochondrial function [80].
Because functional complementation occurs in mitochondrial genomes, it is possible that the presence of functional ETC complexes may phenotypically mask the mtDNA mutational burden of the cell. Therefore, even when the majority of the copies of a particular mitochondrial gene within a single cell harbor a particular mutation, the cell may remain healthy, although eventually clonal expansion or the accumulation of new mutations may lead to a mutational burden that exceeds what the cell can tolerate. This phenotypic threshold is reached when complementation is no longer sufficient to allow sufficient numbers of normal mitochondrial complexes to be formed.

In addition to the clonal expansion of a single mitochondrial mutation, cellular respiration and organismal health may also be affected by the aggregate mutational burden resulting from the net impact of a number of different individually rare mtDNA mutations. For example, 100 different mtDNA mutations, each of which is present in a mitochondrial genome at a frequency of $1 \%$, leads to an aggregate mutational burden of $100 \%$ (meaning an average of 1 mutation per mitochondrial genome). The functional consequences of this situation may be different from the impact of a single specific mutation clonally expanding to reach $100 \%$ (homoplasmy), particularly with respect to the potential role of complementation. Complementation still may allow functional ETC complexes in the case of multiple distinct mutations, whereas this may not be the case for the clonally expanded mutation. This is the case, for example, if the clonally expanded mutation affects a critical amino acid, making it impossible for that cell to make any functional copies of that subunit. Thus, the phenotypic threshold may be much higher for multiple individually rare mutations than for a single clonally expanded mutation.

\section{Somatic mtDNA Mutations in the SN}

The first published study to quantify the number of multiple somatic mtDNA point mutations in the $\mathrm{SN}$ used a highly sensitive cloning and sequencing strategy to characterize the aggregate burden of mtDNA mutations in the frontal cortex and SN of postmortem tissue from PD patients as well as young and old control subjects [71]. This study found that aggregate levels of point mutations increased with age in the frontal cortex of elderly subjects (65-91 years of age) compared to young controls (age 1-24 years). However, this study did not detect a statistically significant increase in the frequency of point mutations in the frontal cortex or $\mathrm{SN}$ of PD patients compared to age-matched controls. The authors hypothesized a number of reasons for this. Firstly, the study used postmortem tissue, which likely represented late stage PD. Therefore, the only dopaminergic neurons included in the analysis were those few that survived to latestage PD when as many as $98 \%$ of the dopaminergic neurons may have already been lost [81]. It is possible that these neurons survived because they were somehow protected from the accumulation of mtDNA mutations. Secondly, these studies used brain tissue (as opposed to individual neurons); therefore the glial contribution may have been increased relative to the neuronal contribution in the PD 
cases because of the scarcity of dopaminergic neurons in late-stages of PD. This could have reduced the relative level of mutations detected in the PD cases as the mean level of mutations is greater in SN neurons compared to glia [82].

To determine whether the frequency of somatic mtDNA point mutations differed between SN neurons and glia, the same authors performed a similarly sensitive cloning and sequencing strategy on laser-capture microdissected single neurons and glial cells from six control subjects [82]. This study did indeed show that the mean level of somatic mtDNA point mutations in single neurons was significantly higher than the mean level in glia with extrapolation of the data suggesting 3.3 somatic point mutations per mitochondrial genome in neurons and 2.2 mutations per mitochondrial genome in glia. In addition, this work demonstrated that heteroplasmy is common in both the D-loop and coding regions even at the level of single neurons and glia. Whether or not somatic mtDNA point mutations reach higher levels in $\mathrm{SN}$ neurons in $\mathrm{PD}$ remains an important question.

\section{ND5 Somatic Mutations in PD}

Other studies have used a similar cloning and sequencing approach to investigate the frequency of mtDNA complex I somatic point mutations in PD patients and aging. One study investigated mutational burden in all seven of the mitochondrial genes encoding the complex I subunits in frontal cortex tissue homogenates [83]. In agreement with previous work [71], the authors found that there was no significant difference in the overall number of point mutations between PD patients and controls. However, this study found that the percentage of transitions was lower than in the previously published work at $39 \%$ in PD and $29 \%$ in controls compared to $88 \%$ of the mutations found in the previous work. The reasons for this apparent discrepancy are not immediately clear. In addition, this work identified low levels of somatic mutations in the ND5 gene that were observed only in PD patients and not seen in controls [83]. Although the same authors subsequently confirmed this finding in another set of PD samples [84], levels of the mutations were quite low, generally less than $1 \%$, well below the levels that would be expected to lead to functional consequences.

\section{0. mtDNA Deletions in SN Neurons in PD and Aging}

In early 2006, back-to-back papers in Nature Genetics reported the first findings on the frequency of mtDNA deletions in human SN neurons $[85,86]$. Both groups used cytochrome $\mathrm{C}$ oxidase (COX) deficiency in SN neurons as a marker of a mitochondrial respiratory chain defect. The Bender et al. study [85] used long-range PCR to amplify mtDNA from COX-deficient and control neurons, and the Kraytsberg et al. study [86] developed a novel singlemolecule PCR technique to quantify the total cellular burden of mtDNA deletions in COX-deficient neurons harboring a mitochondrial respiratory chain defect and control neurons.
Both studies found that mtDNA deletions were present at higher levels in aged controls compared to younger individuals and that these deletions were clonally expanded. In addition, the work from Bender and colleagues identified that mtDNA deletions were higher still in individuals with Parkinson's disease compared to aged controls $(52.3 \%$ and $43.3 \%$ resp.), although the difference was of borderline significance $(P=.06)$.

These findings of surprisingly high levels of clonally expanded mtDNA deletions in SN neurons from elderly subjects, and a correlation of mutations with COX-deficiency, support the hypothesis that the age-related accumulation of somatic mtDNA mutations may contribute to the aging of the human brain and to the pathogenesis of Parkinson's disease. However, because these studies of human samples can be only observational in nature, it is difficult to accrue definitive evidence for functional consequences of mtDNA mutations. For such studies, the POLG "Mutator" mice are informative.

\section{Evidence from the POLG "Mutator" Mice}

The mitochondrial theory of aging proposes that the accumulation of mtDNA mutations lead to the aging process and to age-related neurodegenerative diseases [87]. However, experimental evidence in support of this fundamental theory of aging has been limited. Several years ago, two groups reported that transgenic mice expressing a proofreading deficient form of POLG are phenotypically normal at birth but accumulate very high levels of somatic mtDNA point mutations as they age, resulting in a premature aging phenotype that includes weight loss, reduced subcutaneous fat, hair loss, thinning of bones, anemia, reduced fertility, and early death $[88,89]$. These mice were heralded as evidence in support of the mitochondrial theory of aging. However, it has been argued that POLG mutator mice accumulate mtDNA point mutation levels that are more than an order of magnitude higher than typical levels in aged humans, and so their aging-like phenotypes do not imply that levels of mtDNA mutations achieved during normal aging are functionally relevant [90]. Furthermore, heterozygous POLG mutator mice also accumulate higher mtDNA mutation levels than seen in normal aged mice and yet lack an overt phenotype, leading to further questions as to the relevance of these mice to the normal aging process [91].

In addition, the lack of overt parkinsonism in the POLG mutator mice could be interpreted as arguing against a role for somatic mtDNA mutations in PD. However, there are several important caveats to this argument. It remains possible that a more subtle phenotype may be present in heterozygous POLG mutator mice. For example, testing on behavioral measures sensitive to dopamine deficiency has not been reported. Also, levels of mutation accumulation within individual neurons vary substantially, and a subset of neurons may accumulate levels of mutations during normal aging that cross the phenotypic threshold. Furthermore, the POLG mutator mice are not a good model for assessing the role of mtDNA deletions in humans (see discussion on this issue below). Thus, the role for somatic mtDNA 
mutations during normal aging and in neurodegenerative disease remains an open question.

\section{Point Mutations versus Deletions}

It has been argued that large mtDNA deletions rather than point mutations are the driving force behind the premature aging phenotype in homozygous POLG mutator mice [91]. This argument was based on a "random mutation capture" assay that demonstrated a large-fold relative increase in deletions in the POLG mutator mice, but could not determine the absolute levels of mutations. Other methods revealing the absolute level of deletions revealed that levels of deletions are quite low even in the homozygous mutator mice, especially when compared with the much higher level of point mutations [86, 92]. Moreover, another mouse model, "mito-mice", genetically engineered to accumulate mtDNA deletions, appear healthy without features of premature aging despite reaching deletions in up to $30 \%$ of all mtDNA molecules [93]. Therefore it appears likely that mtDNA point mutations rather than deletions drive the aging phenotype in the POLG mutator mice. In SN neurons from both aged controls and individuals with PD, both point mutations [71, $82]$ and large deletions $[85,86]$ accumulate to high levels. In contrast, as discussed above, in the POLG mutator mice, although point mutations reach high levels, absolute levels of mtDNA deletions remain low. Thus the POLG mutator mice may not be a valid model for understanding the functional impact of mtDNA deletions.

\section{POLG Mutations Lead to Familial Parkinsonism}

First identified as a nuclear encoded protein in 1972, POLG is the sole DNA polymerase in animal mitochondria [94, 95]. Essential for synthesis, replication, and repair of mtDNA, POLG contains three enzymatic activities: a DNA polymerase activity, a $3^{\prime} \rightarrow 5^{\prime}$ exonuclease activity involved in proofreading, and a $5^{\prime}$-deoxyribose phosphate (dRP) lyase activity required for base excision repair [95]. Over 100 pathogenic mutations within the POLG gene have been found to cause a vast array of both neurological and nonneurological disorders including progressive external ophthalmoplegia (PEO), ataxia, and Alpers' syndrome [96]. POLG mutations have been linked to parkinsonism. Clinical assessment of several families with PEO or premature ovarian failure showed significant co of POLG mutations with levodopa responsive parkinsonism, and PET findings were consistent with dopaminergic neuron loss [37, 38, 97]. Two missense mutations (G737R and R853W) in the POLG gene were also detected in two siblings in a family with early-onset familial parkinsonism [98]. Moreover, in a largescale systematic analysis of POLG gene in patients with sporadic idiopathic $\mathrm{PD}$, there is significant clustering of rare variants of the POLG1 CAG-repeat as compared with their matched controls [99]. However, a study conducted on a large number of sporadic PD patients from UK and Italy does not conform to the possibility of linking common
POLG polymorphisms in sporadic PD [100]. Nonetheless, the association of POLG mutations in humans with $\mathrm{SN}$ neuronal loss and parkinsonism, in combination with the premature aging phenotype in the POLG mutator mice, supports the hypothesis that the accumulation of somatic mtDNA point mutations in $\mathrm{SN}$ neurons may be functionally significant and may contribute to the pathogenesis of PD.

\section{Conclusions}

Mitochondrial dysfunction appears to play an important role in the pathogenesis of PD. Although the origin of the mitochondrial dysfunction in PD is unknown, somatic mtDNA point mutations and deletions have been found to accumulate with age and reach high levels in SN neurons. Experimental induction of POLG mutations cause the accumulation of mtDNA mutations leading to a premature aging phenotype in mutator mice. Although overt parkinsonism has not been reported in these mice, mutations in the same gene in humans can lead to dopaminergic dysfunction and parkinsonism. Thus, the accumulation of somatic mtDNA mutations in $\mathrm{SN}$ neurons may contribute to the pathogenesis of PD. This hypothesis, if proven to be correct, implies that strategies to block the accumulation of somatic mtDNA mutations may be protective in PD.

\section{Acknowledgment}

Dr. Simon's laboratory is funded by the NINDS (1R01NS058988 and 1R03AG035223) and by the Michael J. Fox Foundation.

\section{References}

[1] W. D. Parker, S. J. Boyson, and J. K. Parks, "Abnormalities of the electron transport chain in idiopathic Parkinson's disease," Annals of Neurology, vol. 26, no. 6, pp. 719-723, 1989.

[2] A. H. V. Schapira, J. M. Cooper, D. Dexter, P. Jenner, J. B. Clark, and C. D. Marsden, "Mitochondrial complex I deficiency in Parkinson's disease," The Lancet, vol. 1, no. 8649, p. 1269, 1989.

[3] A. H. V. Schapira, I. J. Holt, M. Sweeney, A. E. Harding, P. Jenner, and C. D. Marsden, "Mitochondrial DNA analysis in Parkinson's disease," Movement Disorders, vol. 5, no. 4, pp. 294-297, 1990.

[4] N. Hattori, M. Tanaka, T. Ozawa, and Y. Mizuno, "Immunohistochemical studies on complexes I, II, III, and IV of mitochondria in Parkinson's disease," Annals of Neurology, vol. 30, no. 4, pp. 563-571, 1991.

[5] B. Zheng, Z. Liao, J. J. Locascio et al., "PGC- $1 \alpha$, a potential therapeutic target for early intervention in Parkinson's disease," Science Translational Medicine, vol. 2, no. 52, 2010.

[6] M. Fukuda, C. Edwards, and D. Eidelberg, "Functional brain networks in Parkinson's disease," Parkinsonism and Related Disorders, vol. 8, no. 2, pp. 91-94, 2001.

[7] M. Carbon, M. Felice Ghilardi, V. Dhawan, and D. Eidelberg, "Correlates of movement initiation and velocity in Parkinson's disease: a longitudinal PET study," NeuroImage, vol. 34, no. 1, pp. 361-370, 2007. 
[8] J. W. Langston, P. Ballard, J. W. Tetrud, and I. Irwin, "Chronic parkinsonism in humans due to a product of meperidineanalog synthesis," Science, vol. 219, no. 4587, pp. 979-980, 1983.

[9] R. S. Burns, P. A. LeWitt, and M. H. Ebert, "The clinical syndrome of striatal dopamine deficiency. Parkinsonism induced by 1-methyl-4-phenyl-1,2,3,6-tetrahydropyridine (MPTP)," The New England Journal of Medicine, vol. 312, no. 22, pp. 1418-1421, 1985.

[10] Y. Mizuno, T. Saitoh, and N. Sone, "Inhibition of mitochondrial alpha-ketoglutarate dehydrogenase by 1-methyl-4phenylpyridinium ion," Biochemical and Biophysical Research Communications, vol. 143, no. 3, pp. 971-976, 1987.

[11] R. Betarbet, T. B. Sherer, G. MacKenzie, M. Garcia-Osuna, A. V. Panov, and J. T. Greenamyre, "Chronic systemic pesticide exposure reproduces features of Parkinson's disease," Nature Neuroscience, vol. 3, no. 12, pp. 1301-1306, 2000.

[12] V. Bashkatova, M. Alam, A. Vanin, and W. J. Schmidt, "Chronic administration of rotenone increases levels of nitric oxide and lipid peroxidation products in rat brain," Experimental Neurology, vol. 186, no. 2, pp. 235-241, 2004.

[13] T. B. Sherer, J. R. Richardson, C. M. Testa et al., "Mechanism of toxicity of pesticides acting at complex I: relevance to environmental etiologies of Parkinson's disease," Journal of Neurochemistry, vol. 100, no. 6, pp. 1469-1479, 2007.

[14] M. P. King and G. Attardi, "Human cells lacking mtDNA: repopulation with exogenous mitochondria by complementation," Science, vol. 246, no. 4929, pp. 500-503, 1989.

[15] M. Gu, J. M. Cooper, J. W. Taanman, and A. H. V. Schapira, "Mitochondrial DNA transmission of the mitochondrial defect in Parkinson's disease," Annals of Neurology, vol. 44, no. 2, pp. 177-186, 1998.

[16] R. H. Swerdlow, J. K. Parks, S. W. Miller et al., "Origin and functional consequences of the complex I defect in Parkinson's disease," Annals of Neurology, vol. 40, no. 4, pp. 663-671, 1996.

[17] R. H. Swerdlow, J. K. Parks, D. S. Cassarino et al., "Biochemical analysis of cybrids expressing mitochondrial DNA from Contursi kindred Parkinson's subjects," Experimental Neurology, vol. 169, no. 2, pp. 479-485, 2001.

[18] E. Khusnutdinova, I. Gilyazova, E. Ruiz-Pesini et al., "A mitochondrial etiology of neurodegenerative diseases: evidence from Parkinson's disease," Annals of the New York Academy of Sciences, vol. 1147, pp. 1-20, 2008.

[19] J. M. Shoffner, R. L. Watts, J. L. Juncos, A. Torroni, and D. C. Wallace, "Mitochondrial oxidative phosphorylation defects in Parkinson's disease," Annals of Neurology, vol. 30, no. 3, pp. 332-339, 1991.

[20] C. Huerta, M. G. Castro, E. Coto et al., "Mitochondrial DNA polymorphisms and risk of Parkinson's disease in Spanish population," Journal of the Neurological Sciences, vol. 236, no. 1-2, pp. 49-54, 2005.

[21] E. K. Tan, M. Khajavi, J. I. Thornby, S. Nagamitsu, J. Jankovic, and T. Ashizawa, "Variability and validity of polymorphism association studies in Parkinson's disease," Neurology, vol. 55, no. 4, pp. 533-538, 2000.

[22] R. Egensperger, S. Kösel, N. M. Schnopp, P. Mehraein, and M. B. Graeber, "Association of the mitochondrial tRNA(A4336G) mutation with Alzheimer's and Parkinson's diseases," Neuropathology and Applied Neurobiology, vol. 23, no. 4, pp. 315-321, 1997.
[23] U. Mayr-Wohlfart, G. Rödel, and A. Henneberg, "Mitochondrial tRNA(Gln) and tRNA(Thr) gene variants in Parkinson's disease," European Journal of Medical Research, vol. 2, no. 3, pp. 111-113, 1997.

[24] O. A. Ross, R. McCormack, L. D. Maxwell et al., "mt4216C variant in linkage with the mtDNA TJ cluster may confer a susceptibility to mitochondrial dysfunction resulting in an increased risk of Parkinson's disease in the Irish," Experimental Gerontology, vol. 38, no. 4, pp. 397-405, 2003.

[25] J. Autere, J. S. Moilanen, S. Finnilä et al., "Mitochondrial DNA polymorphisms as risk factors for Parkinson's disease and Parkinson's disease dementia," Human Genetics, vol. 115, no. 1, pp. 29-35, 2004.

[26] A. Pyle, T. Foltynie, W. Tiangyou et al., "Mitochondrial DNA haplogroup cluster UKJT reduces the risk of PD," Annals of Neurology, vol. 57, no. 4, pp. 564-567, 2005.

[27] D. Ghezzi, C. Marelli, A. Achilli et al., "Mitochondrial DNA haplogroup $\mathrm{K}$ is associated with a lower risk of parkinson's disease in Italians," European Journal of Human Genetics, vol. 13, no. 6, pp. 748-752, 2005.

[28] D. K. Simon, S. M. Pulst, J. P. Sutton, S. E. Browne, M. F. Beal, and D. R. Johns, "Familial multisystem degeneration with parkinsonism associated with the 11778 mitochondrial DNA mutation," Neurology, vol. 53, no. 8, pp. 1787-1793, 1999.

[29] D. K. Simon, R. Mayeux, K. Marder, N. W. Kowall, M. F. Beal, and D. R. Johns, "Mitochondrial DNA mutations in complex I and tRNA genes in Parkinson's disease," Neurology, vol. 54, no. 3, pp. 703-709, 2000.

[30] O. Bandmann, M. G. Sweeney, S. E. Daniel, C. D. Marsden, and N. W. Wood, "Mitochondrial DNA polymorphisms in pathologically proven Parkinson's disease," Journal of Neurology, vol. 244, no. 4, pp. 262-265, 1997.

[31] C. Vives-Bauza, A. L. Andreu, G. Manfredi et al., "Sequence analysis of the entire mitochondrial genome in Parkinson's disease," Biochemical and Biophysical Research Communications, vol. 290, no. 5, pp. 1593-1601, 2002.

[32] J. M. Van Der Walt, K. K. Nicodemus, E. R. Martin et al., "Mitochondrial polymorphisms significantly reduce the risk of Parkinson disease," American Journal of Human Genetics, vol. 72, no. 4, pp. 804-811, 2003.

[33] C. Huerta, E. Sánchez-Ferrero, E. Coto et al., "No association between Parkinson's disease and three polymorphisms in the eNOS, nNOS, and iNOS genes," Neuroscience Letters, vol. 413, no. 3, pp. 202-205, 2007.

[34] C. Casali, V. Bonifati, F. M. Santorelli et al., "Mitochondrial myopathy, parkinsonism, and multiple mtDNA deletions in a Sephardic Jewish family," Neurology, vol. 56, no. 6, pp. 802805, 2001.

[35] R. M. Chalmers, M. Brockington, R. S. Howard, B. R. F. Lecky, J. A. Morgan-Hughes, and A. E. Harding, "Mitochondrial encephalopathy with multiple mitochondrial DNA deletions: a report of two families and two sporadic cases with unusual clinical and neuropathological features," Journal of the Neurological Sciences, vol. 143, no. 1-2, pp. 41-45, 1996.

[36] R. Horvath, R. A. Kley, H. Lochmüller, and M. Vorgerd, "Parkinson syndrome, neuropathy, and myopathy caused by the mutation A8344G (MERRF) in tRNALys," Neurology, vol. 68, no. 1, pp. 56-58, 2007.

[37] P. Luoma, A. Melberg, J. O. Rinne et al., "Parkinsonism, premature menopause, and mitochondrial DNA polymerase $\gamma$ mutations: clinical and molecular genetic study," The Lancet, vol. 364, no. 9437, pp. 875-882, 2004. 
[38] A. T. Pagnamenta, J. W. Taanman, C. J. Wilson et al., "Dominant inheritance of premature ovarian failure associated with mutant mitochondrial DNA polymerase gamma," Human Reproduction, vol. 21, no. 10, pp. 2467-2473, 2006.

[39] D. Thyagarajan, S. Bressman, C. Bruno et al., "A novel mitochondrial 12SrRNA point mutation in parkinsonism, deafness, and neuropathy," Annals of Neurology, vol. 48, no. 5, pp. 730-736, 2000.

[40] W. Zheng, K. Khrapko, H. A. Coller, W. G. Thilly, and W. C. Copeland, "Origins of human mitochondrial point mutations as DNA polymerase $\gamma$-mediated errors," Mutation Research, vol. 599, no. 1-2, pp. 11-20, 2006.

[41] K. J. Krishnan, A. K. Reeve, D. C. Samuels et al., "What causes mitochondrial DNA deletions in human cells?" Nature Genetics, vol. 40, no. 3, pp. 275-279, 2008.

[42] A. A. Johnson and K. A. Johnson, "Exonuclease proofreading by human mitochondrial DNA polymerase," The Journal of Biological Chemistry, vol. 276, no. 41, pp. 38097-38107, 2001.

[43] A. A. Johnson and K. A. Johnson, "Fidelity of nucleotide incorporation by human mitochondrial DNA polymerase," The Journal of Biological Chemistry, vol. 276, no. 41, pp. 38090-38096, 2001.

[44] F. M. Yakes and B. Van Houten, "Mitochondrial DNA damage is more extensive and persists longer than nuclear DNA damage in human cells following oxidative stress," Proceedings of the National Academy of Sciences of the United States of America, vol. 94, no. 2, pp. 514-519, 1997.

[45] Y. Kuchino, F. Mori, and H. Kasai, "Misreading of DNA templates containing 8-hydroxydeoxyguanosine at the modified base and at adjacent residues," Nature, vol. 326, no. 6117, pp. 77-79, 1987.

[46] K. C. Cheng, D. S. Cahill, H. Kasai, S. Nishimura, and L. A. Loeb, "8-Hydroxyguanine, an abundant form of oxidative DNA damage, causes $\mathrm{G}-\mathrm{T}$ and $\mathrm{A}-\mathrm{C}$ substitutions," The Journal of Biological Chemistry, vol. 267, no. 1, pp. 166-172, 1992.

[47] P. Mecocci, M. F. Beal, R. Cecchetti et al., "Mitochondrial membrane fluidity and oxidative damage to mitochondrial DNA in aged and AD human brain," Molecular and Chemical Neuropathology, vol. 31, no. 1, pp. 53-64, 1997.

[48] B. Halliwell, "Reactive oxygen species and the central nervous system," Journal of Neurochemistry, vol. 59, no. 5, pp. 16091623, 1992.

[49] P. Jenner and C. W. Olanow, "Oxidative stress and the pathogenesis of Parkinson's disease," Neurology, vol. 47, no. 6, supplement 3, pp. S161-S170, 1996.

[50] J. A. Stuart and M. F. Brown, "Mitochondrial DNA maintenance and bioenergetics," Biochimica et Biophysica Acta, vol. 1757, no. 2, pp. 79-89, 2006.

[51] S. Z. Imam, B. Karahalil, B. A. Hogue, N. C. Souza-Pinto, and V. A. Bohr, "Mitochondrial and nuclear DNA-repair capacity of various brain regions in mouse is altered in an age-dependent manner," Neurobiology of Aging, vol. 27, no. 8, pp. 1129-1136, 2006.

[52] J. M. Shoffner, M. T. Lott, A. S. Voljavec, S. A. Soueidan, D. A. Costigan, and D. C. Wallace, "Spontaneous KearnsSayre/chronic external ophthalmoplegia plus syndrome associated with a mitochondrial DNA deletion: a slip-replication model and metabolic therapy," Proceedings of the National Academy of Sciences of the United States of America, vol. 86, no. 20, pp. 7952-7956, 1989.
[53] M. Hirano, A. Shtilbans, R. Mayeux et al., "Apparent mtDNA heteroplasmy in Alzheimer's disease patients and in normals due to PCR amplification of nucleus-embedded mtDNA pseudogenes," Proceedings of the National Academy of Sciences of the United States of America, vol. 94, no. 26, pp. 1489414899, 1997.

[54] S. Mita, B. Schmidt, E. A. Schon, S. DiMauro, and E. Bonilla, "Detection of 'deleted' mitochondrial genomes in cytochrome-c oxidase-deficient muscle fibers of a patient with Kearns-Sayre syndrome," Proceedings of the National Academy of Sciences of the United States of America, vol. 86, no. 23, pp. 9509-9513, 1989.

[55] J. Muller-Hocker, G. Hubner, K. Bise et al., "Generalized mitochondrial microangiopathy and vascular cytochrome $\mathrm{c}$ oxidase deficiency: occurrence in a case of MELAS syndrome with mitochondrial cardiomyopathy-myopathy and combined complex I/IV deficiency," Archives of Pathology and Laboratory Medicine, vol. 117, no. 2, pp. 202-210, 1993.

[56] E. J. Brierley, M. A. Johnson, R. N. Lightowlers, O. F. W. James, and D. M. Turnbull, "Role of mitochondrial DNA mutations in human aging: implications for the central nervous system and muscle," Annals of Neurology, vol. 43, no. 2, pp. 217-223, 1998.

[57] E. Nekhaeva, N. D. Bodyak, Y. Kraytsberg et al., "Clonally expanded mtDNA point mutations are abundant in individual cells of human tissues," Proceedings of the National Academy of Sciences of the United States of America, vol. 99, no. 8, pp. 5521-5526, 2002.

[58] G. S. Shadel and D. A. Clayton, "Mitochondrial DNA maintenance in vertebrates," Annual Review of Biochemistry, vol. 66, pp. 409-436, 1997.

[59] H. Chen and D. C. Chan, "Mitochondrial dynamics—-fusion, fission, movement, and mitophagy-in neurodegenerative diseases," Human Molecular Genetics, vol. 18, no. 2, pp. R169R176, 2009.

[60] W. M. Brown, M. George, and A. C. Wilson, "Rapid evolution of animal mitochondrial DNA," Proceedings of the National Academy of Sciences of the United States of America, vol. 76, no. 4, pp. 1967-1971, 1979.

[61] J. L. Elson, D. C. Samuels, D. M. Turnbull, and P. F. Chinnery, "Random intracellular drift explains the clonal expansion of mitochondrial DNA mutations with age," American Journal of Human Genetics, vol. 68, no. 3, pp. 802-806, 2001.

[62] H. K. Rajasimha, P. F. Chinnery, and D. C. Samuels, "Selection against pathogenic mtDNA mutations in a stem cell population leads to the loss of the $3243 \mathrm{~A} \rightarrow \mathrm{G}$ mutation in blood," American Journal of Human Genetics, vol. 82, no. 2, pp. 333-343, 2008.

[63] M. Yoneda, A. Chomyn, A. Martinuzzi, O. Hurko, and G. Attardi, "Marked replicative advantage of human mtDNA carrying a point mutation that causes the MELAS encephalomyopathy," Proceedings of the National Academy of Sciences of the United States of America, vol. 89, no. 23, pp. 11164$11168,1992$.

[64] H. A. Coller, N. D. Bodyak, and K. Khrapko, "Frequent intracellular clonal expansions of somatic mtDNA mutations: significance and mechanisms," Annals of the New York Academy of Sciences, vol. 959, pp. 434-447, 2002.

[65] L. Boulet, G. Karpati, and E. A. Shoubridge, "Distribution and threshold expression of the tRNA(Lys) mutation in skeletal muscle of patients with myoclonic epilepsy and ragged-red fibers (MERRF)," American Journal of Human Genetics, vol. 51, no. 6, pp. 1187-1200, 1992. 
[66] W. K. Porteous, A. M. James, P. W. Sheard et al., "Bioenergetic consequences of accumulating the common 4977-bp mitochondrial DNA deletion," European Journal of Biochemistry, vol. 257, no. 1, pp. 192-201, 1998.

[67] R. Rossignol, M. Malgat, J. P. Mazat, and T. Letellier, "Threshold effect and tissue specificity. Implication for mitochondrial cytopathies," The Journal of Biological Chemistry, vol. 274, no. 47, pp. 33426-33432, 1999.

[68] S. Vielhaber, A. Kudin, R. Schröder, C. E. Elger, and W. S. Kunz, "Muscle fibres: applications for the study of the metabolic consequences of enzyme deficiencies in skeletal muscle," Biochemical Society Transactions, vol. 28, no. 2, pp. 159-164, 2000.

[69] A. Nicholas, Y. Kraytsberg, X. Guo, and K. Khrapko, "On the timing and the extent of clonal expansion of mtDNA deletions: evidence from single-molecule PCR," Experimental Neurology, vol. 218, no. 2, pp. 316-319, 2009.

[70] M. T. Lin, D. K. Simon, C. H. Ahn, L. M. Kim, and M. Flint Beal, "High aggregate burden of somatic mtDNA point mutations in aging and Alzheimer's disease brain," Human Molecular Genetics, vol. 11, no. 2, pp. 133-145, 2002.

[71] D. K. Simon, M. T. Lin, L. Zheng et al., "Somatic mitochondrial DNA mutations in cortex and substantia nigra in aging and Parkinson's disease," Neurobiology of Aging, vol. 25, no. 1, pp. 71-81, 2004.

[72] E. A. Schon, S. DiMauro, M. Hirano, and R. W. Gilkerson, "Therapeutic prospects for mitochondrial disease," Trends in Molecular Medicine, vol. 16, no. 6, pp. 268-276, 2010.

[73] G. Attardi, M. Yoneda, and A. Chomyn, "Complementation and segregation behavior of disease-causing mitochondrial DNA mutations in cellular model systems," Biochimica et Biophysica Acta, vol. 1271, no. 1, pp. 241-248, 1995.

[74] M. Satoh and T. Kuroiwa, "Organization of multiple nucleoids and DNA molecules in mitochondria of a human cell," Experimental Cell Research, vol. 196, no. 1, pp. 137-140, 1991.

[75] F. J. Iborra, H. Kimura, and P. R. Cook, "The functional organization of mitochondrial genomes in human cells," BMC Biology, vol. 2, article 9, 2004.

[76] F. Legros, F. Malka, P. Frachon, A. Lombès, and M. Rojo, "Organization and dynamics of human mitochondrial DNA," Journal of Cell Science, vol. 117, no. 13, pp. 2653-2662, 2004.

[77] R. A. Capaldi, R. Aggeler, R. Gilkerson et al., "A replicating module as the unit of mitochondrial structure and functioning," Biochimica et Biophysica Acta, vol. 1555, no. 1-3, pp. 192-195, 2002.

[78] Y. Wang and D. F. Bogenhagen, "Human mitochondrial DNA nucleoids are linked to protein folding machinery and metabolic enzymes at the mitochondrial inner membrane," The Journal of Biological Chemistry, vol. 281, no. 35, pp. 25791-25802, 2006.

[79] R. W. Gilkerson, E. A. Schon, E. Hernandez, and M. M. Davidson, "Mitochondrial nucleoids maintain genetic autonomy but allow for functional complementation," Journal of Cell Biology, vol. 181, no. 7, pp. 1117-1128, 2008.

[80] E. A. Schon and R. W. Gilkerson, "Functional complementation of mitochondrial DNAs: mobilizing mitochondrial genetics against dysfunction," Biochimica et Biophysica Acta, vol. 1800, no. 3, pp. 245-249, 2010.

[81] P. Damier, E. C. Hirsch, Y. Agid, and A. M. Graybiel, “The substantia nigra of the human brain: II. Patterns of loss of dopamine-containing neurons in Parkinson's disease," Brain, vol. 122, no. 8, pp. 1437-1448, 1999.
[82] I. Cantuti-Castelvetri, M. T. Lin, K. Zheng et al., "Somatic mitochondrial DNA mutations in single neurons and glia," Neurobiology of Aging, vol. 26, no. 10, pp. 1343-1355, 2005.

[83] R. Smigrodzki, J. Parks, and W. D. Parker, "High frequency of mitochondrial complex I mutations in Parkinson's disease and aging," Neurobiology of Aging, vol. 25, no. 10, pp. 12731281, 2004.

[84] W. D. Parker and J. K. Parks, "Mitochondrial ND5 mutations in idiopathic Parkinson's disease," Biochemical and Biophysical Research Communications, vol. 326, no. 3, pp. 667-669, 2005.

[85] A. Bender, K. J. Krishnan, C. M. Morris et al., "High levels of mitochondrial DNA deletions in substantia nigra neurons in aging and Parkinson disease," Nature Genetics, vol. 38, no. 5, pp. 515-517, 2006.

[86] Y. Kraytsberg, E. Kudryavtseva, A. C. McKee, C. Geula, N. W. Kowall, and K. Khrapko, "Mitochondrial DNA deletions are abundant and cause functional impairment in aged human substantia nigra neurons," Nature Genetics, vol. 38, no. 5, pp. 518-520, 2006.

[87] A. W. Linnane, S. Marzuki, T. Ozawa, and M. Tanaka, "Mitochondrial DNA mutations as an important contributor to ageing and degenerative diseases," The Lancet, vol. 1, no. 8639, pp. 642-645, 1989.

[88] C. C. Kujoth, A. Hiona, T. D. Pugh et al., "Mitochondrial DNA mutations, oxidative stress, and apoptosis in mammalian aging," Science, vol. 309, no. 5733, pp. 481-484, 2005.

[89] A. Trifunovic, A. Wredenberg, M. Falkenberg et al., "Premature ageing in mice expressing defective mitochondrial DNA polymerase," Nature, vol. 429, no. 6990, pp. 417-423, 2004.

[90] K. Khrapko, Y. Kraytsberg, A. D. N. J. de Grey, J. Vijg, and E. A. Schon, "Does premature aging of the mtDNA mutator mouse prove that mtDNA mutations are involved in natural aging?" Aging Cell, vol. 5, no. 3, pp. 279-282, 2006.

[91] M. Vermulst, J. H. Bielas, G. C. Kujoth et al., "Mitochondrial point mutations do not limit the natural lifespan of mice," Nature Genetics, vol. 39, no. 4, pp. 540-543, 2007.

[92] D. Edgar and A. Trifunovic, "The mtDNA mutator mouse: dissecting mitochondrial involvement in aging," Aging, vol. 1, no. 12, pp. 1028-1032, 2009.

[93] A. Sato, T. Kono, K. Nakada et al., "Gene therapy for progeny of mito-mice carrying pathogenic mtDNA by nuclear transplantation," Proceedings of the National Academy of Sciences of the United States of America, vol. 102, no. 46, pp. 1676516770, 2005.

[94] B. Fridlender, M. Fry, A. Bolden, and A. Weissbach, "A new synthetic RNA-dependent DNA polymerase from human tissue culture cells (HeLa-fibroblast-synthetic oligonucleotidestemplate-purified enzymes)," Proceedings of the National Academy of Sciences of the United States of America, vol. 69, no. 2, pp. 452-455, 1972.

[95] L. S. Kaguni, "DNA polymerase $\gamma$, the mitochondrial replicase," Annual Review of Biochemistry, vol. 73, pp. 293-320, 2004.

[96] L. J. Wong, R. K. Naviaux, N. Brunetti-Pierri et al., "Molecular and clinical genetics of mitochondrial diseases due to POLG mutations," Human Mutation, vol. 29, no. 9, pp. E150E172, 2008.

[97] M. Mancuso, M. Filosto, S. J. Oh, and S. DiMauro, "A novel polymerase $\gamma$ mutation in a family with ophthalmoplegia, neuropathy, and parkinsonism," Archives of Neurology, vol. 61, no. 11, pp. 1777-1779, 2004. 
[98] G. Davidzon, P. Greene, M. Mancuso et al., "Early-onset familial parkinsonism due to POLG mutations," Annals of Neurology, vol. 59, no. 5, pp. 859-862, 2006.

[99] P. T. Luoma, J. Eerola, S. Ahola et al., "Mitochondrial DNA polymerase gamma variants in idiopathic sporadic Parkinson disease," Neurology, vol. 69, no. 11, pp. 1152-1159, 2007.

[100] W. Tiangyou, G. Hudson, D. Ghezzi et al., "POLG1 in idiopathic Parkinson disease," Neurology, vol. 67, no. 9, pp. 1698-1700, 2006. 


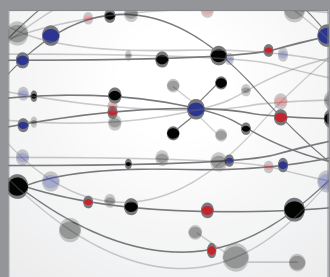

The Scientific World Journal
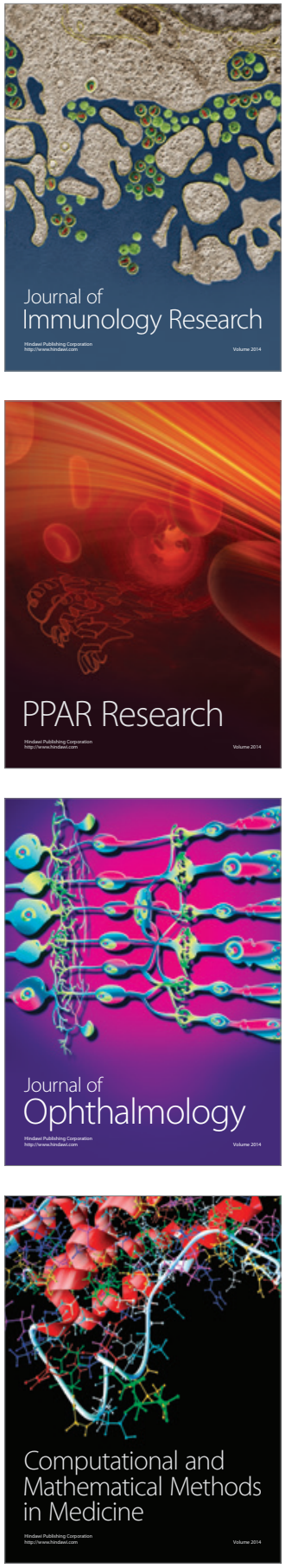

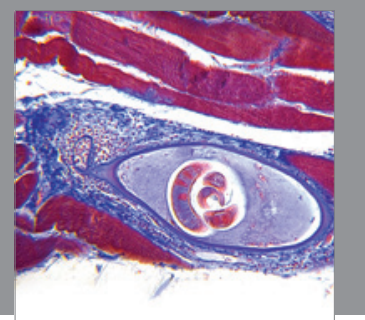

Gastroenterology

Research and Practice
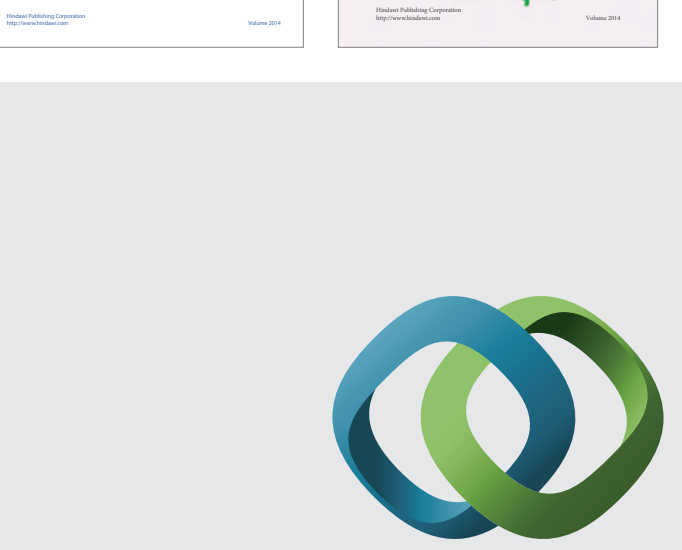

\section{Hindawi}

Submit your manuscripts at

http://www.hindawi.com
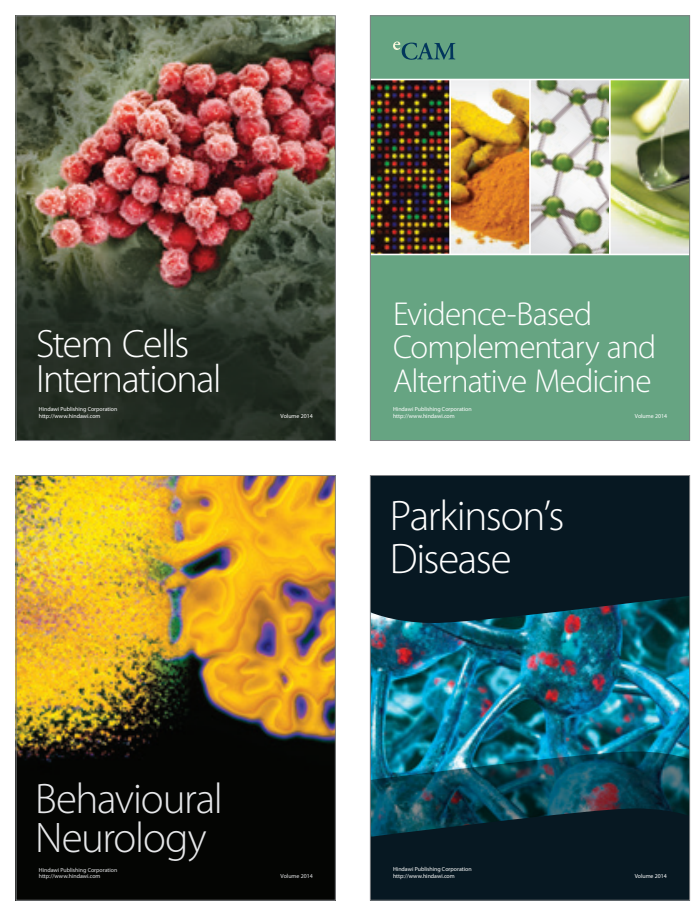

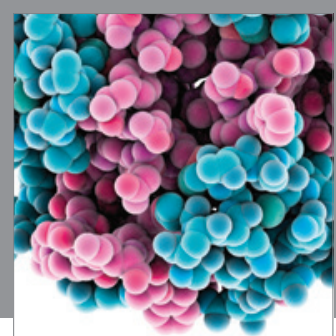

Journal of
Diabetes Research

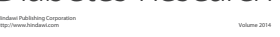

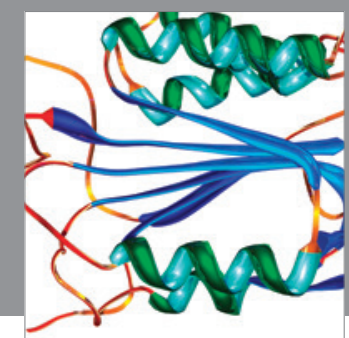

Disease Markers
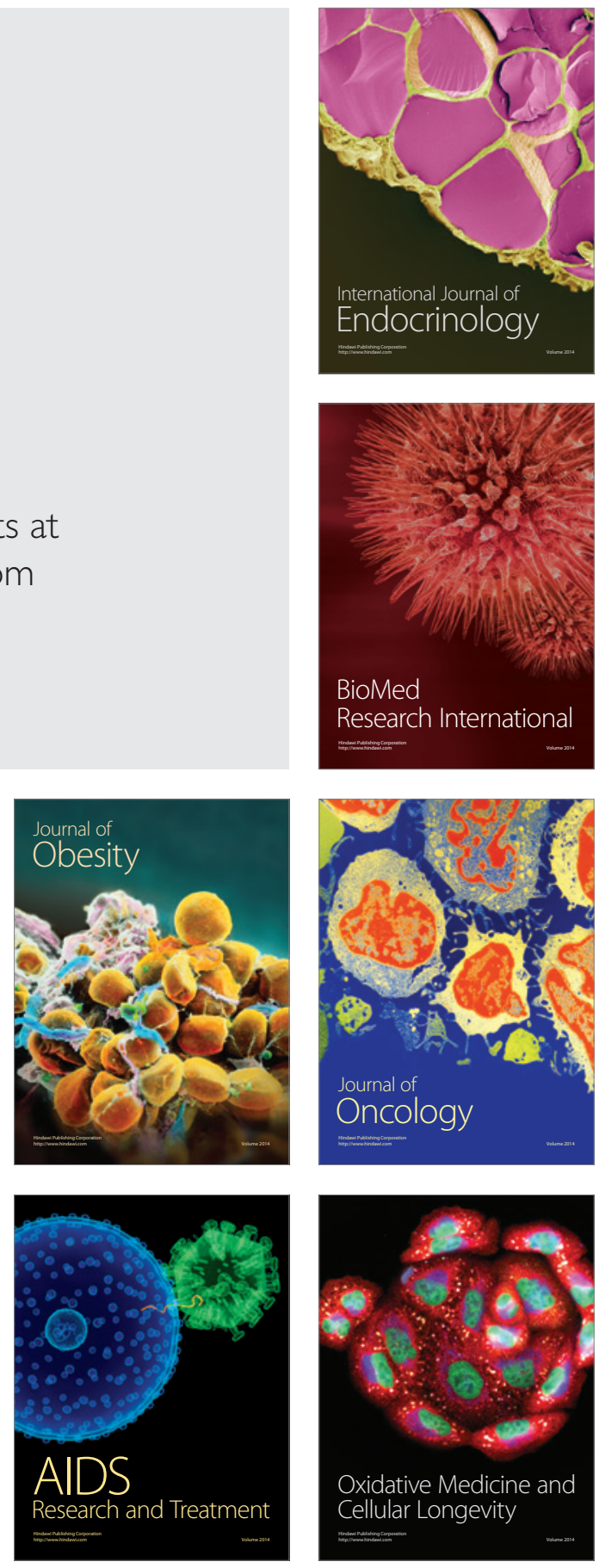